\title{
Consistent Truncation to Three Dimensional (Super-)gravity
}

\author{
Rajesh Kumar Gupta and Ashoke Sen \\ Harish-Chandra Research Institute \\ Chhatnag Road, Jhusi, Allahabad 211019, INDIA \\ E-mail: rajesh@mri.ernet.in, sen@mri.ernet.in, ashokesen1999@gmail.com
}

\begin{abstract}
For a general three dimensional theory of (super-)gravity coupled to arbitrary matter fields with arbitrary set of higher derivative terms in the effective action, we give an algorithm for consistently truncating the theory to a theory of pure (super-)gravity with the gravitational sector containing only Einstein-Hilbert, cosmological constant and Chern-Simons terms. We also outline the procedure for finding the parameters of the truncated theory. As an example we consider dimensional reduction on $S^{2}$ of the 5-dimensional minimal supergravity with curvature squared terms and obtain the truncated theory without any curvature squared terms. This truncated theory reproduces correctly the exact central charge of the boundary CFT.
\end{abstract}




\section{Contents}

1 Introduction

2 Field Redefinition of the Bosonic Fields 3

3 Algorithm for Determining $\Lambda(\phi) \quad 8$

4 Higher Derivative Terms Involving the Gravitino 10

5 Dimensional Reduction of Five Dimensional Supergravity 14

\section{Introduction}

Three dimensional (super-)gravity with negative cosmological constant has played an important role in the study of black holes in string theory [1-3]. The theories relevant for string theory however are not theories of pure (super-)gravity but (super-)gravity coupled to other matter fields containing higher derivative terms. In the absence of other matter fields the higher derivative terms in the action can be removed by field redefinition and the action may be reduced to the standard (super-)gravity action whose gravitational part contains a sum of three terms, the Einstein-Hilbert term, a cosmological constant term and the Chern-Simons term $[4,5]$. An argument based on AdS/CFT correspondence suggests that even when matter fields are present one can carry out a consistent truncation of the theory where only (super-)gravity is present, and action is again that of standard (super-)gravity whose gravitational sector is given by the sum of three terms [6]. The main ingredient of this argument was that in the dual two dimensional (super-)conformal field theory living at the boundary of $A d S_{3}$ any correlation function with one matter field and arbitrary number of (super-)stress tensor vanishes, and furthermore the correlation functions of the (super-)stress tensor are determined completely in terms of the central charge and are independent of the matter content of the theory. One of the goals of the present paper is to describe the consistent truncation procedure directly in the bulk theory without any reference to AdS/CFT correspondence. A general analysis of consistent truncation to supergravity theory in general dimensions can be found in [7-9].

Although our analysis is classical, it can in principle be applied to the full quantum effective action 1 However in our analysis we shall have to assume that the initial action is local, 1.e is

\footnotetext{
${ }^{1}$ If the theory admits an $A d S_{3}$ solution we can define the quantum effective action to be the one whose classical
} 
given by an integral of a local Lagrangian density that admits a derivative expansion. Since in general the full quantum effective action can contain non-local terms, our analysis will not be directly applicable on these terms. In contrast the argument based on AdS/CFT correspondence works for the full quantum corrected effective action.

After consistent truncation and field redefinition that brings the action to the standard form, the parameters labelling the action are the cosmological constant and the coefficient of the Chern-Simons term. Of them the Chern-Simons term does not change under the field redefinition required to bring the action to the standard form but the cosmological constant term is modified. In theories with extended supersymmetry the cosmological constant can be determined from the coefficient of a gauge Chern-Simons terms [10] which also does not get renormalized under the field redefinition; however in general we need to determine the cosmological constant explicitly. We describe a simple algebraic procedure for determining the cosmological constant of the final theory in terms of the parameters of the original action.

Finally we apply our method to the analysis of the three dimensional gravity that arises from the dimensional reduction on $S^{2}$ of five dimensional supergravity with curvature squared corrections [11] and calculate the cosmological constant of the final theory after the field redefinition that brings the action to the standard form. In this case the theory has a $(0,4)$ supersymmetry and the expected value of the cosmological constant can be found by relating it to the coefficient of a gauge Chern-Simons term [10,12]. One can also infer it from the results for the black hole entropy in these theories computed in [13-15]. The result of the explicit calculation agrees with these predictions.

\section{Field Redefinition of the Bosonic Fields}

In this section we shall describe how the bosonic part of a (super-)gravity action coupled to matter fields and containing higher derivative terms can be brought into the form of a standard supergravity action via field redefinition and consistent truncation. We begin with a three dimensional general coordinate invariant theory of gravity coupled to an arbitrary set of matter fields. We denote by $g_{\mu \nu}$ the metric, by $\phi$ the set of all the scalar fields, by $\Sigma$ the set of all other tensor fields, by $R_{\mu \nu}$ the Ricci tensor associated with the metric $g_{\mu \nu}$ and by $R$ the scalar curvature. At the level of two derivative terms, the action takes the form:

$$
S_{0}+S_{\text {matter }},
$$

boundary S-matrix reproduces correctly the full boundary S-matrix of the quantum theory. 
where

$$
S_{0}=\int d^{3} x \sqrt{-g}\left(R+\Lambda_{0}(\phi)\right)
$$

and $S_{\text {matter }}$ denotes the kinetic term for the matter fields. $-\Lambda_{0}(\phi)$ represents the scalar field potential. We have already carried out an appropriate redefinition of the metric to remove a possible $\phi$ dependent function multiplying $R$ in the Einstein-Hilbert term. If $\Lambda_{0}(\phi)$ has an extremum at $\phi=\phi_{0}$ then this theory has a solution where $\phi$ is set equal to $\phi_{0}$, all other tensor fields are set to zero, and the metric is given by that of an $A d S_{3}$ space of size $l_{0}=\sqrt{2 / \Lambda_{0}\left(\phi_{0}\right)}$ for $\Lambda_{0}\left(\phi_{0}\right)>0$ and a $d S_{3}$ space of size $\bar{l}_{0}=\sqrt{-2 / \Lambda_{0}\left(\phi_{0}\right)}$ for $\Lambda_{0}\left(\phi_{0}\right)<0$. In this case $\Lambda_{0}\left(\phi_{0}\right)$ corresponds to the negative of the cosmological constant.

We shall now consider the effect of adding higher derivative terms. For this we shall assume that these terms are small compared to the leading term, in the sense that the length parameter $l_{s}$ that controls these terms is small compared to the length scale $l_{0}$ over which the leading order solution varies 2 We shall also assume that we can associate with each higher derivative term in the Lagrangian density an index $n$ that counts how many powers of $l_{s}$ accompanies this term compared to the leading term. For example if the three dimensional theory is obtained via a dimensional reduction of type IIB string theory on $K 3 \times S^{1} \times S^{2} \times A d S_{3}$ with $K 3$ and $S^{1}$ having size of the order of string scale and $S^{2}$ and $A d S_{3}$ having large size, then $\alpha^{\prime}$ corrections as well as corrections coming from integrating out the heavy modes associated with $K 3 \times S^{1}$ compactification will have index $n>0$, whereas all the terms associated with compactification of supergravity on $S^{2} \times A d S_{3}$ - including the ones involving massive Kaluza-Klein modes will have index 0 . An efficient way to keep track of the derivative expansion is to introduce a derivative counting parameter $\lambda$ and accompany a term of index $n$ by a factor of $\lambda^{n}$. We shall carry out our analysis in a power series expansion in $\lambda$ even though at the end we shall set $\lambda=1$.

Since in three dimension the Riemann tensor $R_{\mu \nu \rho \sigma}$ can be expressed in terms of the Ricci tensor, all the higher derivative terms can be expressed in terms of the Ricci tensor, its covariant derivatives and covariant derivatives of the matter fields. We shall now reorganize these terms as follows. We first note that under $g_{\mu \nu} \rightarrow g_{\mu \nu}+\delta g_{\mu \nu}$,

$$
S_{0} \rightarrow S_{0}-\int d^{3} x \sqrt{-g} P^{\mu v} \delta g_{\mu \nu}+O\left(\delta g^{2}\right),
$$

\footnotetext{
${ }^{2}$ Often the three dimensional theory is obtained from dimensional reduction of a higher dimensional theory on a compact space of size of order $l_{0}$. In this case if we integrate out the Kaluza-Klein modes we shall generate higher derivative terms which are not suppressed by powers of $l_{s}$. To avoid this situation we include all the Kaluza-Klein modes in the set $\Sigma$ without integrating them out.
} 
where

$$
P_{\mu \nu}=R_{\mu \nu}-\frac{1}{2}\left(R+\Lambda_{0}(\phi)\right) g_{\mu \nu}
$$

Defining

$$
P \equiv P_{\mu}^{\mu}=-\frac{1}{2} R-\frac{3}{2} \Lambda_{0}(\phi)
$$

(2.4) can be rewritten as

$$
R_{\mu \nu}=P_{\mu \nu}-\left(P+\Lambda_{0}(\phi)\right) g_{\mu \nu} .
$$

We now eliminate the variables $R_{\mu \nu}, R$ and their covariant derivatives in higher derivative terms by $P_{\mu \nu}, P$ and their covariant derivatives.

In this convention the most general action takes the form: 3

$$
S=S_{0}+\lambda S_{c s}+\widetilde{S}_{\text {matter }}+\lambda^{n} S_{n}
$$

$S_{0}$ is given in (2.2). $\lambda S_{c s}$ is the gravitational Chern-Simons term

$$
S_{c s}=K \int d^{3} x \Omega^{(3)}(\Gamma), \quad \Omega^{(3)}(\Gamma) \equiv \epsilon^{\mu \nu \rho}\left[\frac{1}{2} \Gamma_{\mu \sigma}^{\tau} \partial_{\nu} \Gamma_{\rho \tau}^{\sigma}+\frac{1}{3} \Gamma_{\mu \sigma}^{\tau} \Gamma_{\nu \kappa}^{\sigma} \Gamma_{\rho \tau}^{\kappa}\right],
$$

where $K$ is a constant and $\Gamma_{\nu \rho}^{\mu}$ denotes the Christoffel symbol. Note that we have included a factor of $\lambda$ in $S_{c s}$ since in string theory the gravitational Chern-Simons term typically arises from $\alpha^{\prime}$ corrections. $\widetilde{S}_{\text {matter }}$ denotes the matter terms (including the standard kinetic terms) which are quadratic and higher order in $\Sigma$, derivatives of $\Sigma$ and derivatives of $\phi . \lambda^{n} S_{n}$ denotes all other terms, 1.e. manifestly general coordinate invariant terms up to linear order in $\Sigma, \partial_{\mu} \phi$ and their derivatives, but not terms of the form $\int d^{3} x \sqrt{-g} R f(\phi)$ since they can be included in $S_{0}$. Most general higher derivative terms in the action will have the form given in (2.7) with $n=1$ but for later use we have allowed for the fact that the higher derivative terms which cannot be included in $S_{0}, \widetilde{S}_{\text {matter }}$ or $\lambda S_{c s}$ may actually begin their expansion at order $\lambda^{n}$. It is easy to see that $S_{n}$ must contain least one power of $P_{\mu \nu}$, since the $P_{\mu \nu}$ independent terms which do not involve $\Sigma, \partial_{\mu} \phi$ or their derivatives can be absorbed into $\Lambda_{0}(\phi)$ and $P_{\mu \nu}$ independent terms which are linear in $\Sigma, \partial_{\mu} \phi$ or their derivatives either vanish or become quadratic in $\Sigma$, $\partial_{\mu} \phi$ or their derivatives after integration by parts and hence may be included in $\widetilde{S}_{\text {matter }}$. An alert reader may worry about special cases where a symmetric rank 2 tensor $A_{\mu \nu}$ has a coupling

\footnotetext{
${ }^{3}$ During the process of replacing $R_{\mu \nu}$ by the right hand side of (2.6) we may generate some terms of the form $\int d^{3} x \sqrt{-g} f(\phi)$. Since these cannot be absorbed into $\widetilde{S}_{\text {matter }}$ or $S_{n}$, we need to absorb them into the scalar field potential $\Lambda_{0}(\phi)$ appearing inside $S_{0}$. Thus $\Lambda_{0}(\phi)$ needs to be determined in a self-consistent manner. To any order in power series expansion in $\lambda$ this can be done using an iterative procedure.
} 
proportional to $\sqrt{-g} f_{1}(\phi) g^{\mu v} A_{\mu \nu}$ or an antisymmetric rank three tensor $C_{\mu \nu \rho}$ has a coupling proportional to $f_{2}(\phi) \epsilon^{\mu \nu \rho} C_{\mu \nu \rho}$. We can however avoid these situations by expressing $A_{\mu \nu}$ as $A g_{\mu \nu}+A_{\mu \nu}^{\prime}$ with $A=g^{\mu \nu} A_{\mu \nu} / 3$, and $A_{\mu \nu}^{\prime}$ a traceless symmetric matrix, and $C_{\mu \nu \rho}$ as $C(\sqrt{-g}) \epsilon_{\mu \nu \rho}$ with $C=(\sqrt{-g})^{-1} \epsilon^{\mu \nu \rho} C_{\mu \nu \rho} / 6$, and treating $A$ and $C$ as scalar fields. In this case these terms can be included in the scalar field potential $\Lambda_{0}(\phi)$ appearing in $S_{0}$. Thus $S_{n}$ has the form

$$
S_{n}=\int d^{3} x \sqrt{-g} P^{\mu v} K_{\mu \nu}\left(\phi, \Sigma, \nabla_{\rho}, g_{\rho \sigma}, P_{\rho \sigma}, \lambda\right) .
$$

where $K_{\mu \nu}$ is some combination of matter fields, $P_{\mu \nu}$ and their covariant derivatives, and can contain non-negative powers of $\lambda$.

Now consider a redefinition of the metric of the form

$$
g_{\mu \nu} \rightarrow g_{\mu \nu}+\lambda^{n} K_{\mu \nu}
$$

Under this

$$
\begin{gathered}
S_{0} \rightarrow S_{0}-\lambda^{n} \int d^{3} x \sqrt{-g} P^{\mu v} K_{\mu \nu}+O\left(\lambda^{2 n}\right)=S_{0}-\lambda^{n} S_{n}+O\left(\lambda^{2 n}\right), \\
S_{c s} \rightarrow S_{c s}+O\left(\lambda^{n+1}\right),
\end{gathered}
$$

and

$$
\lambda^{n} S_{n} \rightarrow \lambda^{n} S_{n}+O\left(\lambda^{2 n}\right)
$$

Thus

$$
S_{0}+\lambda S_{c s}+\lambda^{n} S_{n} \rightarrow S_{0}+\lambda S_{c s}+O\left(\lambda^{n+1}\right) .
$$

Furthermore $\widetilde{S}_{\text {matter }}$ remains quadratic in $\Sigma, \partial_{\mu} \phi$ or their derivatives under this field redefinition. The order $\lambda^{n+1}$ term on the right hand side of (2.14) can now be regrouped into a term of the form $\sqrt{-g} f(\phi)$ that can be absorbed into a redefinition of $\Lambda_{0}(\phi)$, a term quadratic in $\Sigma$ and $\partial \phi$ that can be absorbed into $\widetilde{S}_{\text {matter }}(\phi)$ and a term containing at least one power in $P_{\mu \nu}$. Thus the resulting action may be expressed as:

$$
S=S_{0}^{\prime}+\lambda S_{c s}+\widetilde{S}_{\text {matter }}^{\prime}+\lambda^{n+1} S_{n+1},
$$

where

$$
S_{0}^{\prime}=\int d^{3} x \sqrt{-g}\left(R+\Lambda_{0}^{\prime}(\phi)\right)
$$

$\widetilde{S}_{\text {matter }}^{\prime}$ contains terms which are quadratic and higher order in $\Sigma$ and derivatives of $\phi, \Sigma$ and

$$
S_{n+1}=\int d^{3} x \sqrt{-g} P^{\mu v} K_{\mu \nu}^{\prime}\left(\phi, \Sigma, \nabla_{\rho}, g_{\rho \sigma}, P_{\rho \sigma}, \lambda\right)
$$


for some $K_{\mu \nu}^{\prime}$. Thus the new action has the same form as our starting action with $n$ replaced by $n+1$. Repeating this process we can ensure that to any fixed order in an expansion in $\lambda$, the action can be brought to the form:

$$
S=\int d^{3} x \sqrt{-g}(R+\Lambda(\phi))+\lambda S_{c s}+\widetilde{S}_{\text {matter }},
$$

for some choice of $\Lambda(\phi)$ and $\widetilde{S}_{\text {matter }}$.

Now suppose $\Lambda(\phi)$ has an extremum at $\phi=\phi_{0}$. Introducing new fields $\xi=\phi-\phi_{0}$ we may express the action as

$$
S=\int d^{3} x \sqrt{-g}\left(R+\Lambda\left(\phi_{0}\right)\right)+\lambda S_{c s}+\cdots,
$$

where $\cdots$ contain terms which are at least quadratic in $\xi, \Sigma$ and their covariant derivatives. We can now carry out a consistent truncation of the theory by setting $\xi=0, \Sigma=0$. This leaves us with a purely gravitational action with Einstein-Hilbert term, cosmological constant term and Chern-Simons term.

If the theory contains a 2-form field $B$ with gauge invariance $B \rightarrow B+d \Lambda$ then we can consider a slightly more general truncation where instead of setting $B$ to zero we set it to have a constant field strength $C \sqrt{-g} \epsilon_{\mu \nu \rho}$ for some constant $C$. Let $\widetilde{B}$ denote the fluctuation around this fixed background. Since $C \sqrt{-g} \epsilon_{\mu \nu \rho}$ is a general coordinate invariant tensor, and since the Lagrangian density depends on $B$ only through the combination $(d B)_{\mu \nu \rho}=C \sqrt{-g} \epsilon_{\mu \nu \rho}+(d \widetilde{B})_{\mu \nu \rho}$, it depends on $(d \widetilde{B})_{\mu v \rho}$ in a manifestly general coordinate invariant fashion. We can then proceed with our analysis as before, including $\widetilde{B}$ in the list of tensor fields $\Sigma$.

If instead of considering a theory of gravity we consider (extended) supergravity theories, then the theory contains additional fields. In particular the additional bosonic fields in the theory are gauge fields with Chern-Simons terms [16-20]. Thus in order to show that a general higher derivative supersymmetric theory admits a consistent truncation to a supergravity theory we need to show that higher derivative terms involving higher powers of gauge fields can be removed by field redefinition. This follows from the fact that under $A_{\mu} \rightarrow A_{\mu}+\delta A_{\mu}$ the gauge Chern-Simons term changes by a term proportional to $\epsilon^{\mu \nu \rho} \operatorname{Tr}\left(F_{\mu \nu} \delta A_{\rho}\right)$. Thus a term of the form $\lambda^{n} \int \sqrt{-g} \operatorname{Tr}\left(F_{\mu \nu} L^{\mu v}\right)$ in the action may be removed (up to order $\lambda^{2 n}$ terms) by a shift of $A_{\mu}$ proportional to $\sqrt{-g} \epsilon_{\mu \nu \rho} L^{v \rho}$. Following this procedure we can remove all terms involving the gauge fields other than the Chern-Simons term to any order in $\lambda .4$ Once this has been done, one can

\footnotetext{
${ }^{4}$ This assumes that all other terms in the action depend on the gauge field only through $F_{\mu \nu}$ and not explicitly $A_{\mu}$, 1.e. there are no other charged fields on the theory. This is not a restriction on the theory since these charged fields,
} 
then carry out the field redefinition of the metric and the scalar fields as described earlier, and obtain a consistent truncation to a theory of metric and gauge fields with gauge Chern-Simons terms, Einstein-Hilbert term, cosmological constant term and gravitational Chern-Simons term. Supersymmetry then relates the coefficient of the gauge and gravitational Chern-Simons terms to the cosmological constant term.

So far our analysis has been restricted to terms in the action involving bosonic fields only. In a supergravity theory we must also include the fermionic fields and argue that higher derivative terms involving the fermions may be removed by field redefinition. We shall return to this problem in $\$ 4$

\section{Algorithm for Determining $\Lambda(\phi)$}

The analysis of the last section gives an algorithm for carrying out a field redefinition and consistent truncation that gives a theory of pure (super-)gravity. However for any given higher derivative action this is a complicated procedure and one would like to have a simpler algorithm to determine the final truncated theory. Of the various parameters labelling the final theory the coefficients of the Chern-Simons terms are easy to determine since they do not get renormalized from their initial values. On the other hand the cosmological constant term does get renormalized during the field redefinition. In this section we shall outline a simple procedure for finding the exact $\Lambda(\phi)$ appearing in (2.18) without having to carry out all the steps described in the last section. The cosmological constant of the final truncated theory can then be found by determining the value of $\Lambda(\phi)$ at its extremum.

Suppose our initial action has the form

$$
S=\int d^{3} x \sqrt{-g} \mathscr{L}+\lambda S_{c s}
$$

In anticipation of the fact that the final truncation involves setting the scalars $\phi$ to constants and other tensor fields $\Sigma$ to 0 , let us consider a theory of pure gravity obtained by setting $\Sigma$ to 0 and $\phi$ to some constant values in (3.1). Thus $\phi$ can now be regarded as a set of external parameters

if present, can be set to zero in a consistent truncation scheme provided the gauge symmetry is not spontaneously broken. In the latter case the would be Goldstone boson associated with the symmetry breaking would mix with the gauge field via a two point coupling and we cannot have a consistent truncation to pure supergravity. 
labelling the action. We now consider a background

$$
\begin{aligned}
& d s^{2}=-l^{2}\left(1+r^{2}\right) d t^{2}+l^{2}\left(1+r^{2}\right)^{-1} d r^{2}+l^{2} r^{2} d \varphi^{2}, \\
& \phi=\text { constant }, \quad \Sigma=0,
\end{aligned}
$$

representing an $A d S_{3}$ space of size $l$. If we define

$$
F(l, \phi)=l^{3} \mathscr{L}
$$

evaluated in the background (3.2), then the metric satisfies its equation of motion if $l$ is chosen to be at the extremum $l_{\text {ext }}$ of $F$. Furthermore $r F\left(l_{\text {ext }}, \phi\right)$ denotes the value of $\sqrt{-g} \mathcal{L}$ evaluated at the solution. Note that the term in the equations of motion obtained from the variation of the Chern-Simons term automatically vanishes for the $A d S_{3}$ metric (3.2) for any constant $l$.

Let us leave this result aside for a while and consider the form of the action obtained after a field redefinition of the metric as described in $\$ 2$. After setting $\phi$ to a constant and $\Sigma$ to 0 , the action (2.18) takes the form:

$$
S=\int d^{3} x \sqrt{-g}(R+\Lambda(\phi))+\lambda S_{c s}
$$

If we evaluate $\sqrt{-g}(R+\Lambda(\phi))$ for the $A d S_{3}$ background (3.2), we get a new function $r H(l, \phi)$ with

$$
H(l, \phi)=l^{3}\left[-\frac{6}{l^{2}}+\Lambda(\phi)\right] .
$$

Now since we have carried out a field redefinition of the metric but not of $\Sigma$ or $\phi$, we expect $F(l, \phi)$ and $H(l, \phi)$ to be related by a redefinition of the parameter $l$ for any fixed $\phi .5$ Hence the values of these functions at the extremum must be the same. Since the extremum of $H$ occurs at,

$$
\tilde{l}_{\text {ext }}=\sqrt{\frac{2}{\Lambda(\phi)}}, \quad H\left(\tilde{l}_{\text {ext }}, \phi\right)=-\sqrt{\frac{32}{\Lambda(\phi)}},
$$

we get, by setting the right hand side of (3.6) to $F\left(l_{\text {ext }}, \phi\right)$,

$$
\Lambda(\phi)=\frac{32}{F\left(l_{\text {ext }}, \phi\right)^{2}}
$$

\footnotetext{
${ }^{5}$ We are implicitly using the result that during the process of redefinition of the metric the terms arising out of the variation of the Chern-Simons term vanishes when the metric has the form (3.2) and $\partial_{\mu} \phi$ and $\Sigma$ are set to zero. This can be seen from the fact that in this case the field redefinition essentially rescales the metric. Since $\Gamma_{v \rho}^{\mu}$ remains unchanged under a rescaling of the metric and since the Chern-Simons term is constructed entirely in terms of $\Gamma_{v \rho}^{\mu}$, it does not change under such a field redefinition.
} 
provided $F\left(l_{\text {ext }}, \phi\right)$ is negative. This determines $\Lambda(\phi)$.

Eq.(3.7) might give the impression that this procedure always leads to a theory with positive $\Lambda$, 1.e. with a negative cosmological constant. This is however an artifact of the fact that we have already assumed that the theory admits an $A d S_{3}$ solution. It may so happen that $F(l, \phi)$ defined in (3.3) has an extremum at an imaginary value of $l$ and hence $F(l, \phi)$ is imaginary at the extremum. 6 This will give a negative $\Lambda(\phi)$ and hence a positive cosmological constant. A better way to analyze this case is to consider a de Sitter metric of the form

$$
d s^{2}=-\vec{l}^{2}\left(1-r^{2}\right) d t^{2}+\vec{l}^{2}\left(1-r^{2}\right)^{-1} d r^{2}+\vec{l}^{2} r^{2} d \varphi^{2}
$$

instead of the anti-de Sitter metric given in (3.2), and define

$$
\bar{F}(\bar{l}, \phi)=\vec{l}^{3} \mathscr{L},
$$

evaluated in this background with $\phi$ set to constants and $\Sigma$ set to zero. On the other hand (3.5) is now replaced by

$$
\bar{H}(\bar{l}, \phi)=\bar{l}^{3}\left[\frac{6}{\bar{l}^{2}}+\Lambda(\phi)\right] .
$$

and the value of $\bar{H}(\bar{l}, \phi)$ at the extremum with respect to $\bar{l}$ is given by $\sqrt{-32 / \Lambda(\phi)}$. Equating this to the value of $\bar{F}$ at its extremum we get:

$$
\Lambda(\phi)=-\frac{32}{\bar{F}\left(\bar{l}_{\text {ext }}, \phi\right)^{2}}
$$

provided $\bar{F}\left(\bar{l}_{e x t}, \phi\right)$ is positive.

Finally we note that there is always a possibility that neither $F(l, \phi)$ nor $\bar{F}(\bar{l}, \phi)$ has an extremum for real values of $l$ or $\bar{l}$, or even if such extrema exist, the resulting function $\Lambda(\phi)$ does not have an extremum as a function of $\phi$. In this case the theory under consideration does not admit an $A d S_{3}$ or $d S_{3}$ solution and we cannot carry out the consistent truncation following the procedure described above.

\section{Higher Derivative Terms Involving the Gravitino}

In the last two sections we have described how via a field redefinition the bosonic part of the supergravity action can be brought into the standard form. Once the bosonic part of the action

\footnotetext{
${ }^{6}$ Note that the metric and hence $\mathcal{L}$ depends only on $l^{2}$ and hence is real even when $l$ is imaginary.
} 
has been shown to coincide with that of the supergravity action one would expect that supersymmetry will fix the fermionic part of the action uniquely (up to a possible field redefinition involving the fermions) to be that of the standard supergravity action. In this section we shall briefly discuss how such a result might be proven.

We begin with an action where the purely bosonic part has already been brought into the standard form using the field redefinition described in $\$ 2$. At the onset we shall assume that supersymmetry is unbroken at the extremum $\phi_{0}$ of $\Lambda(\phi)$; otherwise we expect the gravitino to mix with the Goldstino and hence the matter and the gravity multiplet will no longer be decoupled. This in turn requires $\Lambda\left(\phi_{0}\right)$ to be positive since we do not have unbroken supersymmetry in de Sitter space. If the theory has altogether $\mathcal{N}$ supersymmetries then there are $\mathcal{N}$ gravitino fields $\psi_{\mu}^{i}$ with $1 \leq i \leq \mathcal{N}$. In the supergravity action of [16-21] the gravitino action has the form:

$$
S_{0}^{\psi}=-\int d^{3} x \epsilon^{\mu \nu \rho} \bar{\psi}_{\mu}^{i} \mathcal{D}_{\nu} \psi_{\rho}^{i},
$$

where

$$
\mathcal{D}_{\mu} \psi_{v}^{i}=\partial_{\mu} \psi_{v}^{i}+\frac{1}{8} \omega_{a b \mu}\left[\gamma^{a}, \gamma^{b}\right] \psi_{\nu}^{i} \pm \sqrt{\frac{\Lambda\left(\phi_{0}\right)}{32}} e_{a \mu} \gamma^{a} \psi_{v}^{i}+A_{\mu}^{a}\left(T^{a}\right)_{i j} \psi_{v}^{j},
$$

$\omega_{\mu a b}$ being the spin connection, $e_{a \mu}$ the vielbeins, $A_{\mu}^{a}$ the gauge fields and $T^{a}$ are the generators of the representation of the gauge group in which the gravitinos transform. The $+(-)$ sign correspond to the gravitinoes associated with left (right) supersymmetries. Under a general variation of the gravitino fields

$$
\delta S_{0}^{\psi}=-\int d^{3} x \epsilon^{\mu \nu \rho}\left[\delta \bar{\psi}_{\mu}^{i} \mathcal{D}_{\nu} \psi_{\rho}^{i}+\text { h.c. }\right]
$$

leading to the gravitino equation of motion

$$
\mathcal{D}_{v} \psi_{\rho}^{i}-\mathcal{D}_{\rho} \psi_{\nu}^{i}=0
$$

The supersymmetry transformation law of the gravitino fields takes the form

$$
\delta_{s} \psi_{\mu}^{i}=\mathcal{D}_{\mu} \epsilon^{i},
$$

where $\epsilon^{i}$ are the supersymmetry transformation parameters.

We shall now examine the possibility of adding higher derivative terms in the action and also possibly in the supersymmetry transformation laws. Let us denote by $\eta$ the set of all the bosonic and fermionic fields coming from the matter sector with the scalars measured relative 
to $\phi_{0}$ (1.e. the set $\eta$ contains the shifted fields $\xi$ introduced above (2.19)). A higher derivative term in the action which is quadratic or higher order in $\eta$ is harmless since we can consistently truncate the theory by setting $\eta=0$. Thus we need to worry about terms which are at most linear in $\eta$ or derivatives of $\eta$. We shall refer to these as the dangerous terms since, if present, they will prevent us from consistently truncating the theory to the one described by the standard supergravity action. As in $\$ 2$ we shall organise these terms according to the power of the derivative counting parameter $\lambda$ that they carry. Let us suppose that the first dangerous higher derivative terms in the Lagrangian density appear at order $\lambda^{k}$. Now any term that is proportional to the equation of motion of the metric, the gauge fields or the gravitinos derived from the leading supergravity action can be absorbed into a redefinition of these fields at the cost of generating higher order terms; thus we need to look for terms which do not vanish identically when leading order supergravity equations of motion are satisfied. Using this we can remove all the dangerous terms in the action which contain any power of gauge field strength, the combination $R_{\mu \nu}+\Lambda(\phi) g_{\mu \nu}$, and commutators of covariant derivatives. Thus the dangerous terms may be expressed as general coordinate invariant and local Lorentz invariant combinations of the gravitino fields, their symmetrized covariant derivatives and the metric. We now consider all the order $\lambda^{k}$ dangerous terms and organise them by their rank, - defined as the total power of $\psi_{\mu}$ and $\bar{\psi}_{\mu}$ contained in that term. We begin with the terms of lowest rank, - call it $m_{0} . m_{0}$ cannot vanish since we have already argued earlier that all the dangerous terms without the gravitino field can be removed by field redefinition. (For this we need to include in the set $\Sigma$ of $\S 2$ all the matter fermions as well.) For non-zero $m_{0}$ the lowest order supersymmetry variation of the gravitino described in (4.5) has the effect of producing a term of rank $\left(m_{0}-1\right)$, constructed out of the gravitino fields, their symmetrized covariant derivatives, the metric, and covariant derivatives of the supersymmetry transformation parameter. In order for supersymmetry to be preserved, such terms need to be cancelled by some other terms. The terms arising from the supersymmetry variation of the bosons in the original rank $m_{0}$ term are of rank $\geq m_{0}$ and hence cannot cancel the rank $\left(m_{0}-1\right)$ term. Thus there are two possibilities: 1$)$ the rank $\left(m_{0}-1\right)$ terms arising from the variation of the gravitino cancel among themselves after we integrate by parts and move all the derivatives from $\epsilon, \bar{\epsilon}$ to the fields, possibly after modifying the supersymmetry transformation laws of the supergravity fields, and 2) we can try to cancel these terms against terms coming from supersymmetry variation of the bosons in a term of rank $\left(m_{0}-2\right)$. Of these the first possibility would mean that the dangerous terms are invariant under the transformation (4.5) of the gravitino alone up to terms which vanish by lowset order supergravity equations 
of motion.7 To see if this is possible we first focus on the terms with maximum number of derivatives where all the covariant derivatives have been replaced by ordinary derivatives in the order $\lambda^{k}$, rank $m_{0}$ term in the action. The net supersymmetry variation of these terms under the supersymmetry transformation law (4.5) must vanish after using the lowest order gravitino equations of motion (4.4) with $\mathcal{D}_{\mu}$ replaced by $\partial_{\mu}$ in (4.5) and (4.4), since this is the term in $\delta_{s} S$ with maximum number of derivatives at this order. In this case the gravitino satisfying its lowest order equations of motion has the form $\psi_{\mu}^{i}=\partial_{\mu} \chi^{i}, \bar{\psi}_{\mu}^{i}=\partial_{\mu} \bar{\chi}^{i}$ for some $\chi^{i}, \bar{\chi}^{i}$. Let us evaluate the order $\lambda^{k}$, rank $m_{0}$ term in the action in this background. By assumption the result is not identically zero, - otherwise we could have removed these terms from the action by a field redefinition of the gravitino field. Now for $\psi_{\mu}^{i}=\partial_{\mu} \chi^{i}, \bar{\psi}_{\mu}^{i}=\partial_{\mu} \bar{\chi}^{i}$ the gauge transformation laws of the gravitino field take the form $\chi^{i} \rightarrow \chi^{i}+\epsilon^{i}, \bar{\chi}^{i} \rightarrow \bar{\chi}^{i}+\bar{\epsilon}^{i}$. Since $\epsilon^{i}$ and $\bar{\epsilon}^{i}$ can be taken to be independent parameters we consider a situation where only one of the $\epsilon^{i}$ is not zero. Invariance under supersymmetry transformation then tells us that the term under consideration is invariant under $\chi^{i} \rightarrow \chi^{i}+\epsilon^{i}$ for an arbitrary function $\epsilon^{i}$. In other words the term is independent of $\chi^{i}$. Repeating this argument we conclude that the term under consideration must be independent of all $\chi^{i}$ and $\bar{\chi}^{i}$. Thus it must vanish since it vanishes when we set all the $\chi^{i}$ and $\bar{\chi}^{i}$ to zero. This contradicts our original assertion that the term does not vanish identically. This leads us to the conclusion that the original order $\lambda^{k}$, rank $m_{0}$ term in the action, with covariant derivatives replaced by ordinary derivatives, must have been such that after suitable integration by parts and commutation of the derivative operators it vanishes when the gravitino satisfies its lowest order equation of motion.

How does the conclusion change when the ordinary derivatives are replaced by covariant derivatives? Since we know that the term can be manipulated and shown to vanish when covariant derivatives are replaced by ordinary derivatives, we can carry out the same manipulation. The only possible extra terms which could arise must be proportional to the commutators $\left[D_{\mu}, D_{v}\right]$ since the covariant derivatives can be manipulated in the same manner as the ordinary derivatives except for their commutators. However these commutators can be reduced to terms with lower number of derivatives using the lowest order metric and gauge field equations of motion. We can now repeat our analysis on these left-over terms with lower number of derivatives and show that they must be further reducible to terms with lower number of derivatives.

\footnotetext{
${ }^{7}$ The terms proportional to the lowest order equations of motion of the supergravity fields can be cancelled by modifying the supersymmetry transformation laws of the supergravity fields, since the additional variation of the lowest order supergravity action under the modified supersymmetry transformation laws will be a linear combination of the lowest order equations of motion of these fields.
} 
Repeating this procedure we can show that a term that is invariant under the lowest order supersymmetry transformation of the gravitino alone, must vanish as a consequence of lowest order supergravity field equations, and hence can be removed by a field redefinition.

We now turn to the second possibility. This requires the action to contain higher derivative terms of order $\lambda^{k}$ and rank $\left(m_{0}-2\right)$. Since by assumption the action does not contain any dangerous term of rank $\left(m_{0}-2\right)$ to order $\lambda^{k}$, the only possibility is to try to generate these terms from the supersymmetry variation of a non-dangerous term of rank $\left(m_{0}-2\right)$. In order to rule out this possibility we need to make one assumption: as a consequence of unbroken supersymmetry the matter sector fields transform to terms which contain at least a single power of the matter sector field, 1.e. we have $\delta_{s} \eta \sim O(\eta) .8$ In this case terms quadratic and higher order in $\eta$ transform to terms quadratic and higher order in $\eta$ and cannot cancel terms which are at most linear in $\eta$. This rules out the last possibility. Thus we see that it is not possible to add higher derivative dangerous terms in the action in a manner consistent with supersymmetry.

\section{Dimensional Reduction of Five Dimensional Supergravity}

In this section we shall consider five dimensional supergravity with curvature squared term coupled to a set of vector multiplets [11] and dimensionally reduce this theory on $S^{2}$ in the presence of background magnetic flux through $S^{2}$ to get a three dimensional $(0,4)$ supergravity with curvature squared term, coupled to a set of matter fields. We then apply the procedure of $\$ 2$ and $\$ 3$ to truncate this to a pure supergravity theory with gravitational Chern-Simons term, but no other higher derivative terms.

We shall concentrate our attention on the part of the action involving the bosonic fields only. In the three dimensional theory this involves the metric and an $\mathrm{SU}(2)$ gauge field that arises during the dimensional reduction of the five dimensional theory on $S^{2}$. As we have seen at the end of $\$ 2$, reducing the gauge field action to pure Chern-Simons term is relatively simple; hence we shall focus on the part of the action involving the metric. For this we can restrict the fields to the SU(2) invariant sector from the beginning. Since the SU(2) R-symmetry of the three dimensional supergravity can be identified with the rotational symmetry of the compact $S^{2}$, this allows us to carry out the dimensional reduction by restricting the field configurations to rotationally invariant form 9

\footnotetext{
${ }^{8}$ This is of course true at the lowest order in $\lambda$ but we shall assume that this property continues to hold even after including possible higher derivative corrections to the supersymmetry transformation laws.

${ }^{9}$ One might worry about the extra terms which may be generated during the redefinition of the gauge field that
} 
The five dimensional $\mathcal{N}=2$ supergravity has a Weyl multiplet, a set of vector multiplets and a compensator hypermultiplet. After gauge fixing to Poincare supergravity, the bosonic fields of the theory include the metric $g_{a b}$, the two-form auxiliary field $v_{a b}$, a scalar auxiliary field $D$, a certain number $\left(n_{V}\right)$ of one-form gauge fields $A_{a}^{I}$ with $1 \leq I \leq n_{V}$, and an equal number of scalars $M^{I}$ [11]. Here $a, b, .$. are five dimensional coordinate labels and run from 0 to 4 . We shall denote by $F^{I}=d A^{I}$ the field strength associated with the gauge field $A^{I}$. The action for bosonic fields including curvature squared terms can be written as

$$
S=\frac{1}{4 \pi^{2}} \int d^{5} x \sqrt{-g^{(5)}}\left[\mathscr{L}_{0}+\mathscr{L}_{1}\right]
$$

where $\mathscr{L}_{0}$ is the lagrangian at two derivative order and $\mathscr{L}_{1}$ denotes the supersymmetric completion of the curvature squared terms. The explicit forms of $\mathcal{L}_{0}$ and $\mathcal{L}_{1}$ are $[11,14]$

$$
\begin{gathered}
\mathscr{L}_{0}=-2\left(\frac{1}{4} D-\frac{3}{8} R-\frac{1}{2} v^{2}\right)+N\left(\frac{1}{2} D+\frac{1}{4} R+3 v^{2}\right)+2 N_{I} v^{a b} F_{a b}^{I} \\
+N_{I J}\left(\frac{1}{4} F_{a b}^{I} F^{J a b}+\frac{1}{2} \partial_{a} M^{I} \partial^{a} M^{J}\right)+\frac{1}{24} e^{-1} c_{I J K} A_{a}^{I} F_{b c}^{J} F_{d e}^{K} \epsilon^{a b c d e} \\
\mathscr{L}_{1}=\frac{c_{2 I}}{24}\left[\frac{1}{16} e^{-1} \epsilon_{a b c d e} A^{I a} C^{b c f g} C_{f g}^{d e}+\frac{1}{8} M^{I} C^{a b c d} C_{a b c d}+\frac{1}{12} M^{I} D^{2}+\frac{1}{6} F^{I a b} v_{a b} D\right. \\
-\frac{1}{3} M^{I} C_{a b c d} v^{a b} v^{c d}-\frac{1}{2} F^{I a b} C_{a b c d} v^{c d}+\frac{4}{3} M^{I} \nabla^{a} v^{b c} \nabla_{a} v_{b c}+\frac{4}{3} M^{I} \nabla^{a} v^{b c} \nabla_{b} v_{c a} \\
+\frac{8}{3} M^{I}\left(v_{a b} \nabla^{b} \nabla_{c} v^{a c}+\frac{2}{3} v^{a c} v_{c b} R_{a}^{b}+\frac{1}{12} v^{a b} v_{a b} R\right)-\frac{2}{3} e^{-1} M^{I} \epsilon_{a b c d e} v^{a b} v^{c d} \nabla_{f} v^{e f} \\
+\frac{2}{3} e^{-1} F^{I a b} \epsilon_{a b c d e} v^{c f} \nabla_{f} v^{d e}+e^{-1} F^{I a b} \epsilon_{a b c d e} v_{f}^{c} \nabla^{d} v^{e f}-\frac{4}{3} F^{I a b} v_{a c} v^{c d} v_{d b} \\
\left.-\frac{1}{3} F^{I a b} v_{a b} v^{2}+4 M^{I} v_{a b} v^{b c} v_{c d} v^{d a}-M^{I}\left(v_{a b} v^{a b}\right)^{2}\right]
\end{gathered}
$$

where $c_{I J K}$ and $c_{2 I}$ are parameters of the theory, $e \equiv \sqrt{-g}$, and

$$
\begin{gathered}
N=\frac{1}{6} c_{I J K} M^{I} M^{J} M^{K} \\
N_{I}=\frac{1}{2} c_{I J K} M^{J} M^{K} \\
N_{I J}=c_{I J K} M^{K},
\end{gathered}
$$

brings the gauge field action into the standard form; however one can easily argue that these terms cannot affect the final form of the action involving the metric since setting all $S U(2)$ non-invariant fields, including the gauge fields, to zero provides a consistent truncation of the theory. 
and $C_{a b c d}$ is the Weyl tensor defined as

$$
C_{c d}^{a b}=R_{c d}^{a b}+\frac{1}{6} R \delta_{[c}^{[a} \delta_{d]}^{b]}-\frac{4}{3} \delta_{[c}^{[a} R_{d]}^{b]}
$$

The parameters $c_{2 I}$ appear in the coefficients of the higher derivative terms; thus we can keep track of the derivative expansion by simply counting the power of $c_{2 I}$ appearing in the various terms.

We now carry out the dimensional reduction on $S^{2}$ and focus on the sector invariant under the $S O(3)$ isometry group of $S^{2}$. This can be done using the following ansatz for the five dimensional fields

$$
\begin{aligned}
& d s^{2}=g_{\mu \nu}^{(3)}(x) d x^{\mu} d x^{\nu}+\chi^{2}(x) d \Omega^{2}, \quad 0 \leq \mu, \nu \leq 2 \\
& v_{\theta \phi}=V(x) \sin \theta \\
& F_{\theta \phi}^{I}=\frac{p^{I}}{2} \sin \theta, \quad F_{\mu \nu}^{I}=\partial_{\mu} A_{\nu}^{I}-\partial_{\nu} A_{\mu}^{I},
\end{aligned}
$$

with the mixed components of $F_{a b}^{I}$ and $v_{a b}$ set to zero. Here $x^{\mu}$ denote the three dimensional coordinates. All the scalar fields can be arbitrary functions of $x$ but are independent of the coordinates $(\theta, \phi)$ of $S^{2}$. For the metric given in (5.8) the non-vanishing components of the Riemann tensor are

$$
\begin{gathered}
R_{\mu \nu \sigma \rho}=R_{\mu \nu \sigma \rho}^{(3)}, \quad R_{i \mu j v}=-\chi^{-1} g_{i j} \nabla_{\mu} \nabla_{\nu} \chi, \quad R_{i j k l}=\chi^{-2}\left(g_{i k} g_{j l}-g_{i l} g_{j k}\right)\left(1-g^{(3) \mu v} \partial_{\mu} \chi \partial_{\nu} \chi\right) \\
0 \leq \mu, v \leq 2, \quad i, j=\theta, \phi
\end{gathered}
$$

Here $R_{\mu \nu \rho \sigma}^{(3)}$ is the Riemann tensor and $\nabla_{\mu}$ is the covariant derivative computed using the three dimensional metric $g_{\mu \nu}^{(3)}$. Using these relations we get the dimensionally reduced action to be

$$
\begin{aligned}
S= & -\frac{c_{2} \cdot p}{96 \pi} \int d^{3} x \Omega^{(3)}(\Gamma) \\
& +\int d^{3} x \sqrt{-g^{(3)}} \frac{\chi^{2}}{\pi}\left(\frac{3}{4}+\frac{1}{4} N+\frac{c_{2} \cdot M}{288} \frac{1}{\chi^{2}}+\frac{c_{2} \cdot M}{72} \frac{V^{2}}{\chi^{4}}-\frac{c_{2} \cdot p}{288} \frac{V}{\chi^{4}}\right) R^{(3)} \\
& +\int d^{3} x \sqrt{-g^{(3)}} \frac{\chi^{2}}{\pi} U\left(\chi, M^{I}, V, p^{I}, D\right) \\
& +\int d^{3} x \sqrt{-g^{(3)}} \frac{\chi^{2}}{\pi} \frac{c_{2} \cdot M}{192}\left(\frac{8}{3} R_{\mu \nu}^{(3)} R^{(3) \mu \nu}-\frac{5}{6} R^{(3) 2}+\frac{16}{3 \chi} R_{\mu \nu}^{(3)} \nabla^{\mu} \nabla^{v} \chi-\frac{4}{3 \chi} R^{(3)} \nabla^{2} \chi\right) \\
& +\int d^{3} x \sqrt{-g^{(3)}} \widehat{\mathscr{L}\left(\chi, v_{\mu \nu}, M^{I}, F_{\mu \nu}^{I}, R_{\mu \nu}^{(3)}\right)}
\end{aligned}
$$


where

$$
\begin{aligned}
U\left(\chi, M^{I}, V, p^{I}, D\right)= & \frac{2}{\chi^{2}}\left(\frac{3}{4}+\frac{1}{4} N\right)-2\left(\frac{1}{4} D-\frac{V^{2}}{\chi^{4}}\right)+N\left(\frac{1}{2} D+\frac{6 V^{2}}{\chi^{4}}\right) \\
& +\frac{2(N \cdot p) V}{\chi^{4}}+\frac{N_{I J} p^{I} p^{J}}{8 \chi^{4}}+\frac{c_{2} \cdot M}{96 \chi^{4}}+\frac{c_{2} \cdot M}{288} D^{2}+\frac{c_{2} \cdot p}{144} \frac{V D}{\chi^{4}} \\
& -\frac{5}{36}\left(c_{2} \cdot M\right) \frac{V^{2}}{\chi^{6}}-\frac{c_{2} \cdot p}{48} \frac{V}{\chi^{6}}+\frac{c_{2} \cdot p}{36} \frac{V^{3}}{\chi^{8}}+\frac{c_{2} \cdot M}{6} \frac{V^{4}}{\chi^{8}}
\end{aligned}
$$

and $\widehat{\mathscr{L}}\left(\chi, v_{\mu \nu}, M^{I}, F_{\mu \nu}^{I}, R_{\mu \nu}^{(3)}\right)$ denotes terms which are at least quadratic in $\nabla_{\mu} \chi, v_{\mu \nu}, \nabla_{\mu} M^{I}$ and $F_{\mu \nu}^{I}$. In eq.(5.10) all covariant derivatives are computed using the three dimensional metric $g_{\mu \nu}^{(3)}$.

We first need to redefine our metric in such a manner that the coefficient of $R^{(3)}$ in the second line of the action (5.10) can be absorbed into the metric. We define

$$
\widetilde{g}_{\mu \nu}=\psi^{-2} g_{\mu \nu}^{(3)}
$$

where

$$
\psi^{-1}=\frac{\chi^{2}}{\pi}\left(\frac{3}{4}+\frac{1}{4} N+\frac{c_{2} \cdot M}{288} \frac{1}{\chi^{2}}+\frac{c_{2} \cdot M}{72} \frac{V^{2}}{\chi^{4}}-\frac{c_{2} \cdot p}{288} \frac{V}{\chi^{4}}\right)
$$

After substituting (5.12) into the action (5.10), we get

$$
\begin{aligned}
S= & -\frac{c_{2} \cdot p}{96 \pi} \int d^{3} x \Omega^{(3)}(\widetilde{\Gamma}) \\
& +\int d^{3} x \sqrt{-\widetilde{g}}\left[\widetilde{R}+Z\left(\chi, M^{I}, V, p^{I}, D\right)\right] \\
& +\int d^{3} x \sqrt{-\widetilde{g}} \frac{\chi^{2}}{\pi \psi} \frac{c_{2} \cdot M}{192}\left(\frac{8}{3} \widetilde{R}^{\mu \nu} \widetilde{R}_{\mu \nu}-\frac{16}{3 \psi} \widetilde{R}^{\mu \nu} \widetilde{\nabla}_{\mu} \widetilde{\nabla}_{\nu} \psi+\frac{4}{3 \psi} \widetilde{R}^{2} \psi-\frac{5}{6} \widetilde{R}^{2}+\frac{16}{3 \chi} \widetilde{R}^{\mu \nu} \widetilde{\nabla}_{\mu} \widetilde{\nabla}_{v} \chi-\frac{4}{3 \chi} \widetilde{R}^{2} \chi\right) \\
& +\int d^{3} x \sqrt{-\widetilde{g}} \widehat{\mathscr{L}}^{\prime}\left(\chi, v_{\mu \nu}, M^{I}, F_{\mu \nu}^{I}, \widetilde{R}_{\mu \nu}\right)
\end{aligned}
$$

where

$$
Z\left(\chi, M^{I}, V, p^{I}, D\right)=\psi^{3} \frac{\chi^{2}}{\pi} U\left(\chi, M^{I}, V, p^{I}, D\right)
$$

and $\widehat{\mathcal{L}}^{\prime}$ denotes terms quadratic and higher order in the derivatives of scalar fields and other tensor fields. For shorthand notation we denote all scalar fields by $\phi$ i.e. $\left(\chi, M^{I}, V, p^{I}, D\right) \equiv \phi$.

Following the general procedure given in $\$ 2$ we now define

$$
\begin{aligned}
& P_{\mu \nu}=\widetilde{R}_{\mu \nu}-\frac{1}{2} \widetilde{g}_{\mu \nu}\left[\widetilde{R}+\Lambda_{0}(\phi)\right] \\
& P=-\frac{1}{2} \widetilde{R}-\frac{3}{2} \Lambda_{0}(\phi),
\end{aligned}
$$


where $\Lambda_{0}(\phi)$ is a function to be determined later, and rewrite the action as

$$
\begin{aligned}
S= & -\frac{c_{2} \cdot p}{96 \pi} \int d^{3} x \Omega^{(3)}(\widetilde{\Gamma})+\int d^{3} x \sqrt{-\widetilde{g}}[\widetilde{R}+Z(\phi)]+\int d^{3} x \sqrt{-\widetilde{g}} P_{\mu \nu} K^{\mu \nu} \\
& +\int d^{3} x \sqrt{-\widetilde{g}} \frac{\chi^{2}}{\psi \pi} \frac{c_{2} \cdot M}{384} \Lambda_{0}^{2}(\phi) \\
& +\int d^{3} x \sqrt{-\widetilde{g}} \widetilde{\mathscr{L}}
\end{aligned}
$$

where

$$
\begin{aligned}
K_{\mu \nu}= & \frac{\chi^{2}}{\psi \pi} \frac{c_{2} \cdot M}{192}\left[\frac{8}{3} P_{\mu \nu}-\frac{2}{3} \widetilde{g}_{\mu \nu} P+\frac{2}{3} \widetilde{g}_{\mu \nu} \Lambda_{0}(\phi)-\frac{16}{3 \psi} \widetilde{\nabla}_{\mu} \widetilde{\nabla}_{\nu} \psi\right. \\
& \left.+\frac{8}{3 \psi} \widetilde{g}_{\mu \nu} \widetilde{\nabla}^{2} \psi+\frac{16}{3 \chi} \widetilde{\nabla}_{\mu} \widetilde{\nabla}_{\nu} \chi-\frac{8}{3 \chi} \widetilde{g}_{\mu \nu} \widetilde{\nabla}^{2} \chi\right]
\end{aligned}
$$

and $\widetilde{\mathcal{L}}$ denotes terms quadratic and higher order in the derivatives of the scalar fields and other tensor fields. We now choose $\Lambda_{0}(\phi)$ to be the solution to the equation

$$
\Lambda_{0}(\phi)=Z(\phi)+\frac{\chi^{2}}{\psi \pi} \frac{c_{2} \cdot M}{384} \Lambda_{0}(\phi)^{2},
$$

so that the action (5.17) may be expressed as

$$
\begin{aligned}
S= & -\frac{c_{2} \cdot p}{96 \pi} \int d^{3} x \Omega^{(3)}(\widetilde{\Gamma})+\int d^{3} x \sqrt{-\widetilde{g}}\left[\widetilde{R}+\Lambda_{0}(\phi)\right]+\int d^{3} x \sqrt{-\widetilde{g}} P_{\mu \nu} K^{\mu \nu} \\
& +\int d^{3} x \sqrt{-\widetilde{g}} \widetilde{\mathscr{L}} .
\end{aligned}
$$

In this case, as we mentioned earlier, the required field redefinition which will remove the four derivative terms from the action (5.20) is

$$
\widetilde{g}_{\mu v} \rightarrow \widetilde{g}_{\mu v}+K_{\mu v}
$$

To this order the scalar field potential $-\Lambda(\phi)$ is given by

$$
\Lambda(\phi)=\Lambda_{0}(\phi)=Z(\phi)+\frac{\chi^{2}}{\psi \pi} \frac{c_{2} \cdot M}{384} Z^{2}(\phi)+O\left(c_{2}^{2}\right) .
$$

This process can now be repeated to remove the six and higher derivative terms from the action, but we shall not go through the details of the analysis. Our interest is in finding the exact expression for $\Lambda(\phi)$ since this is what controls the final truncated action. We have already described the algotithm for finding $\Lambda(\phi)$ in $\$ 3$. The first step is to compute $F(l, \phi)$ for the 
action (5.14) by evaluating the Lagrangian density (without the Chern-Simons term) in the $A d S_{3}$ background (3.2) with constant scalar fields and vanishing tensor fields. We get

$$
F(l, \phi)=-6 l+l^{3} Z(\phi)+2 a \frac{1}{l}
$$

where

$$
a=\frac{\chi^{2}}{\psi \pi} \frac{c_{2} \cdot M}{192} .
$$

The extremum of $F(l, \phi)$ with respect to $l$ occurs at 10

$$
l_{\text {ext }}^{2}=\frac{1}{Z(\phi)}+\frac{1}{Z(\phi)} \sqrt{1+\frac{2 a}{3} Z(\phi)} \text {. }
$$

Hence $\Lambda(\phi)$ is given by

$$
\Lambda(\phi)=\frac{32}{F\left(l_{\text {ext }}, \phi\right)^{2}}=\frac{32 Z(\phi)}{W(\phi)}\left(2 a \frac{Z(\phi)}{W(\phi)}+W(\phi)-6\right)^{-2}, \quad W(\phi) \equiv 1+\sqrt{1+\frac{2 a}{3} Z(\phi)} .
$$

Before we proceed we note that to order $c_{2 I}$ terms, 1.e. order $a$ term, eq.(5.26) reduces to

$$
\Lambda(\phi)=Z(\phi)+\frac{1}{2} a Z(\phi)^{2}+O\left(a^{2}\right) .
$$

This agrees with the result (5.22) of the explicit calculation to this order.

We now return to the full expression (5.26) for $\Lambda(\phi)$. $\Lambda(\phi)$ has an extremum at the supersymmetric attractor point $[13,14]$

$$
\begin{aligned}
& \chi=\frac{p b}{2} \\
& M^{I}=\frac{p^{I}}{p b} \\
& V=-\frac{3 p b}{8} \\
& D=\frac{12}{p^{2} b^{2}}
\end{aligned}
$$

where

$$
p^{3} \equiv \frac{1}{6} c_{I J K} p^{I} p^{J} p^{K}, \quad b^{3}=1+\frac{c_{2} \cdot p}{12 p^{3}}
$$

\footnotetext{
${ }^{10}$ There is, in principle, another extremum at $l_{\text {ext }}^{2}=(Z(\phi))^{-1}(1-\sqrt{1+2 a Z(\phi) / 3})$. This could in principle describe a de Sitter solution. However since for this solution $\left|l_{\text {ext }}\right| \sim a$, the radius is small and there is no systematic derivative expansion.
} 
The value of $\Lambda(\phi)$ at it's extremum is given by

$$
\Lambda\left(\phi_{0}\right)=\frac{32 \pi^{2}}{p^{6}}\left[1+\frac{c_{2} \cdot p}{8 p^{3}}\right]^{-2}
$$

Thus the final truncated theory, obtained by setting $\phi$ to its value at the extremum and other matter fields to zero, is given by

$$
S=\int d^{3} x \sqrt{-\widetilde{g}}\left(\widetilde{R}+\Lambda\left(\phi_{0}\right)\right)-\frac{c_{2} \cdot p}{96 \pi} \int d^{3} x \Omega^{(3)}(\widetilde{\Gamma}) .
$$

From this one can compute the central charges of the conformal field theory living on boundary of AdS using standard formulæ (see e.g. [6]). The result is

$$
\begin{aligned}
& c_{L}=24 \pi\left(\sqrt{\frac{2}{\Lambda\left(\phi_{0}\right)}}-\frac{c_{2} \cdot p}{96 \pi}\right)=6 p^{3}+\frac{1}{2} c_{2} \cdot p \\
& c_{R}=24 \pi\left(\sqrt{\frac{2}{\Lambda\left(\phi_{0}\right)}}+\frac{c_{2} \cdot p}{96 \pi}\right)=6 p^{3}+c_{2} \cdot p
\end{aligned}
$$

These results agree with the predictions of $[10,12]$ from the requirement of $(0,4)$ supersymmetry, as well as the explicit calculations of [13-15] from the computation of the black hole entropy.

\section{References}

[1] K. Sfetsos and K. Skenderis, "Microscopic derivation of the Bekenstein-Hawking entropy formula for non-extremal black holes," Nucl. Phys. B 517, 179 (1998) [arXiv:hep-th/9711138].

[2] A. Strominger, "Black hole entropy from near-horizon microstates," JHEP 9802, 009 (1998) [arXiv:hep-th/9712251].

[3] K. Skenderis, "Black holes and branes in string theory," Lect. Notes Phys. 541, 325 (2000) [arXiv:hep-th/9901050].

[4] E. Witten, “(2+1)-Dimensional Gravity as an Exactly Soluble System,” Nucl. Phys. B 311, 46 (1988).

[5] E. Witten, “Three-Dimensional Gravity Revisited," arXiv:0706.3359 [hep-th]. 
[6] J. R. David, B. Sahoo and A. Sen, " $A d S_{3}$, Black Holes and Higher Derivative Corrections," JHEP 0707, 058 (2007) [arXiv:0705.0735 [hep-th]].

[7] M. J. Duff and C. N. Pope, "Consistent Truncations In Kaluza-Klein Theories," Nucl. Phys. B 255, 355 (1985).

[8] C. N. Pope and K. S. Stelle, "Zilch Currents, Supersymmetry And Kaluza-Klein Consistency,” Phys. Lett. B 198, 151 (1987).

[9] J. P. Gauntlett and O. Varela, "Consistent Kaluza-Klein Reductions for General Supersymmetric AdS Solutions," arXiv:0707.2315 [hep-th].

[10] P. Kraus and F. Larsen, "Microscopic black hole entropy in theories with higher derivatives," JHEP 0509, 034 (2005) [arXiv:hep-th/0506176].

[11] K. Hanaki, K. Ohashi and Y. Tachikawa, "Supersymmetric Completion of an $R^{2}$ Term in Five-Dimensional Supergravity," Prog. Theor. Phys. 117, 533 (2007) [arXiv:hep-th/0611329].

[12] P. Kraus and F. Larsen, "Holographic gravitational anomalies," JHEP 0601, 022 (2006) [arXiv:hep-th/0508218].

[13] A. Castro, J. L. Davis, P. Kraus and F. Larsen, "5D attractors with higher derivatives," JHEP 0704, 091 (2007) [arXiv:hep-th/0702072].

[14] A. Castro, J. L. Davis, P. Kraus and F. Larsen, "5D Black Holes and Strings with Higher Derivatives,” JHEP 0706, 007 (2007) [arXiv:hep-th/0703087].

[15] M. Alishahiha, "On $R^{2}$ corrections for 5D black holes," arXiv:hep-th/0703099.

[16] A. Achucarro and P. K. Townsend, "A CHERN-SIMONS ACTION FOR THREEDIMENSIONAL ANTI-DE SITTER SUPERGRAVITY THEORIES,” Phys. Lett. B 180, 89 (1986).

[17] A. Achucarro and P. K. Townsend, "EXTENDED SUPERGRAVITIES IN d $=(2+1)$ AS CHERN-SIMONS THEORIES,” Phys. Lett. B 229, 383 (1989).

[18] J. de Boer, "Six-dimensional supergravity on $\mathrm{S} * * 3 \times \operatorname{AdS}(3)$ and $2 \mathrm{~d}$ conformal field theory," Nucl. Phys. B 548, 139 (1999) [arXiv:hep-th/9806104]. 
[19] M. Nishimura and Y. Tanii, "Super Weyl anomalies in the AdS/CFT correspondence," Int. J. Mod. Phys. A 14, 3731 (1999) [arXiv:hep-th/9904010].

[20] J. R. David, "Anti-de Sitter gravity associated with the supergroup $\mathrm{SU}(1,1-2) \times$ SU(1,1-2)," Mod. Phys. Lett. A 14, 1143 (1999) [arXiv:hep-th/9904068].

[21] A. Giacomini, R. Troncoso and S. Willison, "Three-dimensional supergravity reloaded," arXiv:hep-th/0610077. 